\title{
Mutant TDP-43 in motor neurons promotes the onset and progression of ALS in rats
}

\author{
Cao Huang, ${ }^{1}$ Jianbin Tong, ${ }^{1}$ Fangfang Bi, ${ }^{1}$ Hongxia Zhou, ${ }^{1,2}$ and Xu-Gang Xia1 \\ 'Department of Pathology, Anatomy and Cell Biology, and 'Department of Neurology, Thomas Jefferson University, Philadelphia, Pennsylvania, USA.
}

\begin{abstract}
Amyotrophic lateral sclerosis (ALS) is characterized by progressive motor neuron degeneration, which ultimately leads to paralysis and death. Mutation of TAR DNA binding protein 43 (TDP-43) has been linked to the development of an inherited form of ALS. Existing TDP-43 transgenic animals develop a limited loss of motor neurons and therefore do not faithfully reproduce the core phenotype of ALS. Here, we report the creation of multiple lines of transgenic rats in which expression of ALS-associated mutant human TDP-43 is restricted to either motor neurons or other types of neurons and skeletal muscle and can be switched on and off. All of these rats developed progressive paralysis reminiscent of ALS when the transgene was switched on. Rats expressing mutant TDP-43 in motor neurons alone lost more spinal motor neurons than rats expressing the disease gene in varying neurons and muscle cells, although these rats all developed remarkable denervation atrophy of skeletal muscles. Intriguingly, progression of the disease was halted after transgene expression was switched off; in rats with limited loss of motor neurons, we observed a dramatic recovery of motor function, but in rats with profound loss of motor neurons, we only observed a moderate recovery of motor function. Our finding suggests that mutant TDP-43 in motor neurons is sufficient to promote the onset and progression of ALS and that motor neuron degeneration is partially reversible, at least in mutant TDP-43 transgenic rats.
\end{abstract}

\section{Introduction}

Amyotrophic lateral sclerosis (ALS) is characterized by progressive degeneration of motor neurons and denervation atrophy of skeletal muscles. The disease inexorably progresses to paralysis and to death, often within 5 years after diagnosis. While ubiquitinated TAR DNA binding protein 43 (TDP-43) inclusion is a characteristic of sporadic ALS, pathogenic mutation of TDP-43 causes an inherited form of ALS $(1,2)$. Forced expression of either WT or mutant TDP-43 in mice and rats causes compound phenotypes, including cortical dementia and paralysis (3-9). Cortical pathology in transgenic animals is expected, as TDP-43 proteinopathy is identified in sporadic frontotemporal lobe dementia (FTLD) $(2,10)$. TDP-43 is highly conserved and is tightly regulated $(7,11)$. Overexpression of human TDP-43 in mice deregulates the mouse TDP-43, and this interference may be pathogenic (7). However, forced expression of WT mouse TDP-43 causes similar pathology in mice (9), suggesting that increased expression of normal TDP-43 is neurotoxic. As a ribonucleoprotein (12), TDP-43 binds to both DNA and RNA sequences of multiple genes $(13,14)$. Alteration in TDP43 expression interrupts mRNA processing of a number of genes $(15,16)$. While the nature of TDP-43 mutation remains unclear, mutation or deregulation of TDP-43 may affect multiple cellular functions. Animal models that have been reported overexpress TDP-43 restrictedly in the forebrain or globally in all the tissues and thus develop limited or no loss of motor neurons (3-9), insufficiently reproducing the core phenotype of ALS.

ALS is currently incurable because the most effective drug, riluzole, only extends a patient's life by several months. In the SOD1 transgenic model of ALS, $\beta$-lactam antibiotics stimulate the expression of the glutamine transporter and produce a therapeutic effect (17). Knocking down the expression of mutant SOD1

Authorship note: Cao Huang and Jianbin Tong contributed equally to this work. Conflict of interest: The authors have declared that no conflict of interest exists. Citation for this article: J Clin Invest. 2012;122(1):107-118. doi:10.1172/JCI59130. by RNAi produces a therapeutic effect, but does not prevent the disease from progression in transgenic mice (18-20). In the development of effective therapies for ALS, a compelling need is to determine whether ALS could be prevented from progression at certain disease stages. Motor neurons may undergo retrograde degeneration in ALS, and the motor axon terminals are considered the primary targets of degeneration in $\operatorname{ALS}(3,21)$. It is not known whether a functional recovery could be obtained at early disease stages and how functional recovery is related to the integrity of motor circuitry in ALS.

To induce neurodegenerative diseases, disease factors may not be required to be present in all neurons and glia, although unhealthy cells may interact aberrantly with neighboring cells to initiate or to propagate neurodegeneration. Overexpression of mutant buntingtin in multiple types of neurons, but not in cortical pyramidal neurons alone, causes cortical neuropathology in mice (22). Neurons of the same type may degenerate in distinctive ways in response to mutation of different genes. Purkinje neurons degenerate cell autonomously in response to mutation of the npc1 gene (23), but they degenerate non-cell autonomously in response to mutation of the ataxia-7 gene (24). In mice, restricted overexpression of mutant SOD1 in motor neurons does not cause neuron death (25), although mutant SOD1 in neurons is a primary determinant of disease onset (26). While forced expression of normal SOD1 in mice fails to induce ALS phenotypes (27), overexpression of normal TDP-43 causes profound neurodegeneration $(7,9)$. In addition, TDP-43 proteinopathy is not observed in ALS caused by mutation in sod1 (28). Mutant SOD1 and TDP-43 may cause one disease by varied mechanisms. How motor neurons degenerate (cell autonomously or non-cell autonomously) in response to TDP-43 mutation remains to be determined.

Using a Tet-regulatory system, we created multiple lines of transgenic rats expressing mutant human TDP-43 reversibly and restrictedly in motor neurons or in varying types of neurons and skeletal muscles. Overexpression of mutant TDP-43 in neurons 
A

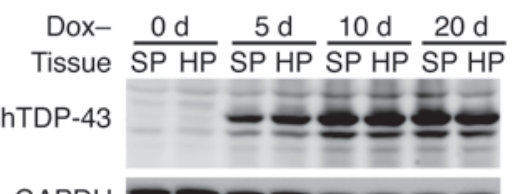

GAPDH

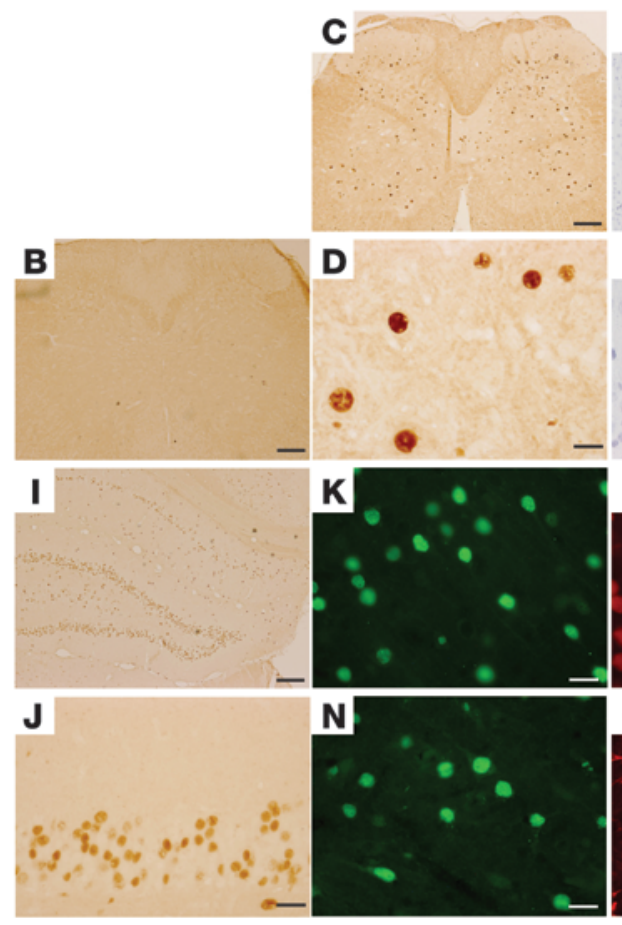

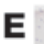

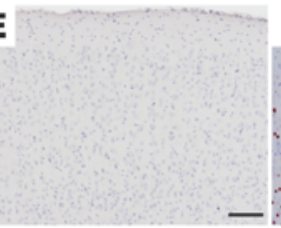

F
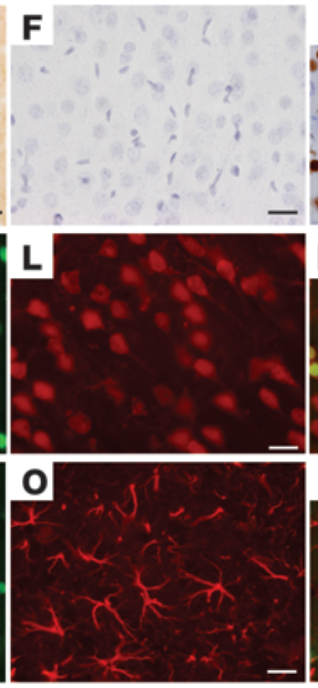

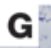

G
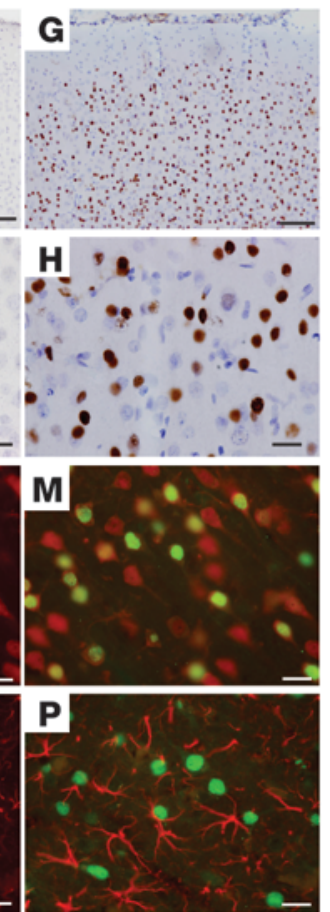

\section{Figure 1}

Restricted overexpression of mutant human TDP-43 (hTDP-43) in neurons is achieved in rats. (A) Immunoblotting showed that the hTDP-43 transgene was expressed in the spinal cord (SP) and hippocampus (HP) of NEF-tTA/TRETDP-43 ${ }^{\mathrm{M} 37 \mathrm{~V}}$ double-transgenic rats soon after Dox was withdrawn (Dox-). The rats were deprived of Dox at the age of 60 days. Equal loading was confirmed by probing the same membrane with an antibody to GAPDH. (B-J) Human TDP43 immunoreactivity was examined in the spinal cord (B-D), cortex (E-H), and hippocampus (I and J) of NEF-tTA/TRETDP-43M337V double-transgenic (C, D, and $\mathbf{G}-\mathbf{J}$ ) and NEF-tTA single-transgenic $(\mathbf{B}, \mathbf{E}$, and $\mathbf{F})$ rats. Coronal sections of neocortex were counterstained lightly with hematoxylin to display the nuclei (E-H). (K-P) Double-label fluorescence staining revealed that human TDP-43 (green, $\mathbf{K}, \mathbf{M}, \mathbf{N}$, and $\mathbf{P}$ ) was colocalized with the neuronal marker NeuN (red, $\mathbf{L}$ and $\mathbf{M}$ ), but was not colocalized with the astrocyte marker GFAP (red: $\mathbf{O}$ and P) in the cortex. Rats were examined 10 days after Dox withdrawal. Scale bars: $200 \mu \mathrm{m}$ (B, C, and I); $100 \mu \mathrm{m}$ (E and $\mathbf{G}) ; 20 \mu \mathrm{m}(\mathbf{D}, \mathbf{F}, \mathbf{H}$, and $\mathbf{J}-\mathbf{P})$. and in muscles caused progressive paralysis in rats, with moderate loss of spinal motor neurons, whereas restricted overexpression of mutant TDP-43 in motor neurons alone caused progressive paralysis in rats, with profound loss of spinal motor neurons. We treated paralyzed rats with doxycycline (Dox) to suppress further expression of the mutant TDP-43 after disease onset and observed a recovery of motor function in the transgenic rats. Functional recovery was moderate in the rats with profound loss of motor neurons, but it was dramatic in paralyzed rats with limited loss of motor neurons. Our findings suggest that motor neuron degeneration can be stopped from progression and that functional recovery is correlated with the integrity of motor neurons.

\section{Results}

Overexpression of mutant TDP-43 in neurons and muscles causes progressive paralysis in rats. We previously showed that ubiquitous overexpression of mutant TDP-43 in rats causes early onset and rapid progression of paralysis reminiscent of ALS (3). To improve the rat model, we intended to overexpress mutant TDP-43 selectively in neurons and chose the regulatory elements (promoter) of the human neurofilament heavy chain $(N E F)$ gene to direct transgene expression (Supplemental Figure 1; supplemental material available online with this article; doi:10.1172/JCI59130DS1). In our TDP-43 transgenic rats $(3,29)$, tetracycline response element (TRE) drives TDP-43 transgene, whose expression fully depends on the activity of tetracycline-controlled transactivator ( $\mathrm{tTA})$. A short fragment $(8.5 \mathrm{~kb})$ of human NEF promoter synthesizes transgene in differentiated neurons in mice (30). We used a long fragment $(18 \mathrm{~kb})$ of the NEF promoter to drive tTA transgene in rats. We established a single-copy transgenic line that exhibits $\mathrm{X}$ chromosome-linked transmission of the NEF-tTA transgene (Supplemental Figure 1). To avoid gene insertion-caused mutation, we only used female rats heterozygous for NEF-tTA in the following studies. By crossing NEF-tTA transgene onto a TRE-TDP-43 ${ }^{\mathrm{M} 337 \mathrm{~V}}$ transgenic background (line 16), we detected a substantial Dox-regulated expression of human TDP-43 transgene (Figure 1, A-J). Expression of the transgene was restricted in neurons (Figure 1, K-M) and was not detected in astrocytes (Figure 1, N-P) - the largest population of nonneuronal cells in the CNS. Unexpectedly, skeletal muscles also expressed human TDP-43 transgene (Supplemental Figure 1).

To induce disease phenotypes in adult rats, we deprived NEF-tTA/ TRE-TDP- $43^{\mathrm{M} 337 \mathrm{~V}}$ double-transgenic rats of Dox at the age of 60 days and observed a full activation of mutant TDP-43 gene in rats by the age of 70 days (Figure 1A). Two weeks after mutant TDP-43 was expressed, the mutant rats began losing grip strength and mobility (Figure 2, A and B), displaying the early signs of paralysis (disease onset). Within 3 weeks after mutant TDP-43 activation, rats lost the ability to retract hind legs (Figure 2, A-C, and Supplemental Video 1). To accurately assess motor neuron death in rats, we used unbiased stereological cell counting. In our publications $(3,31)$, we counted the number of motor neurons in 1 spinal segment (L3). To increase the accuracy of cell counting, here we counted the number of motor neurons in a long segment of lumbar cords (L3-L5). Stereological cell counting revealed a moderate, but significant, loss of neurons in the spinal cord and dentate gyrus of transgenic rats at disease end stages (Figure 2, D-K). In mutant 

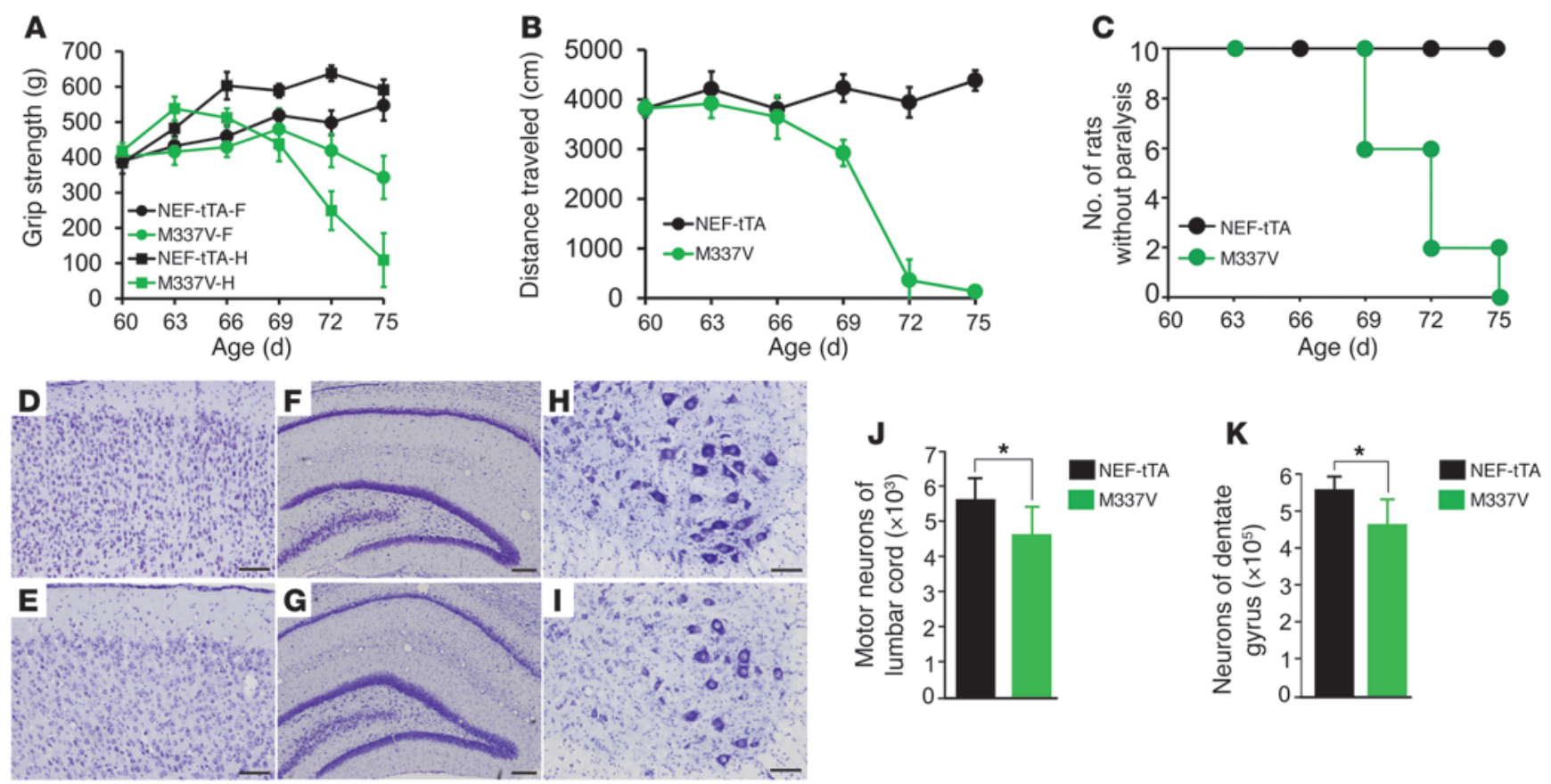

\section{Figure 2}

Overexpression of mutant TDP-43 in neurons causes progressive paralysis in rats. (A) The grip strength of forepaws (f) and hind paws (h) was measured for NEF-tTA/TRE-TDP-43M337V double-transgenic (M337V) and NEF-tTA single-transgenic rats. Data are means \pm SD $(n=7)$. (B) Open-field assay revealed an unrecoverable reduction of mobility in M337V rats after TDP-43 transgene was turned on. Data are means \pm SD $(n=7)$. (C) M337V rats rapidly developed paralysis after the transgene was turned on. Within 2 to 3 days, disease progressed from paralysis stage to end stages. (D-I) Cresyl violet staining revealed the structure of frontal cortex ( $\mathbf{D}$ and $\mathbf{E})$, hippocampus (F and $\mathbf{G})$, and the ventral horn of the lumbar spinal cord $(\mathbf{H}$ and $\mathbf{I})$. The tissues were taken from M337V rats at disease end stages $(\mathbf{E}, \mathbf{G}$, and $\mathbf{I})$ or from NEF-tTA rats at matched ages ( $\mathbf{D}, \mathbf{F}$, and $\mathbf{H})$. ( $\mathbf{J}$ and $\mathbf{K}$ ) Stereological cell counting revealed the number of motor neurons ( $>25 \mu \mathrm{m}$ in diameter) in lumbar cords (L3-L5) and revealed the number of neurons in dentate gyrus. Data are means $\pm \mathrm{SD}(n=5)$. ${ }^{*} P<0.05$. All rats used were females and were constantly given Dox in drinking water $(50 \mu \mathrm{g} / \mathrm{ml})$ until they were 60 days old. Scale bars: $100 \mu \mathrm{m}(\mathbf{D}-\mathbf{E}, \mathbf{H}$, and I); $200 \mu \mathrm{m}(\mathbf{F}$ and $\mathbf{G})$.

TDP-43 transgenic rats at paralysis stages, toluidine blue staining and electromicroscopy revealed degenerating axons in the ventral, but not in the dorsal, roots (Figure 3, K-N, and Supplemental Figure 2, C and D). Silver staining revealed degenerating neurons in the ventral horn of the lumbar cord (Supplemental Figure 2, A and B). At paralysis stages, mutant TDP-43 transgenic rats displayed grouped atrophy of skeletal muscles revealed by H\&E staining (Figure 3B) and by histochemistry for nonspecific esterase (Figure $3 \mathrm{E}$ ) and ATPase (Figure 3H). In contrast, NEF-tTA single-transgenic rats did not display any of these pathological changes (Figures 2 and 3; Supplemental Figure 2). Restricted overexpression of mutant TDP-43 in neurons and in muscles induced motor neuron degeneration and denervation atrophy of muscles, reproducing the key features of ALS in rats.

Ubiquitinated TDP-43 inclusion is a characteristic of sporadic ALS and FTLD (2). We next examined TDP-43 inclusion, but did not observe typical TDP-43 inclusion in mutant TDP-43 transgenic rats at paralysis stages (Figure $1, \mathrm{D}$ and $\mathrm{H}$ ). We detected rare ubiquitin aggregates in the cortex (Supplemental Figure 3), but not in the spinal cord (data not shown), of paralyzed rats. Our finding confirmed that TDP-43 inclusion is not essential to neuron death, at least in rodent models $(3,7)$.

Removal of mutant TDP-43 partly restores motor function in ALS rats expressing the disease gene in neurons and muscles. Restricted expression of mutant TDP-43 in neurons and in muscles caused severe damage to motor axons and to skeletal muscles (Figure 3 and Supplemental Figure 2), but only caused a moderate loss of motor neurons in the spinal cord (Figure 2). We determined the potential of functional recovery in the diseased rats. Since expression of mutant TDP-43 in rats was subject to Dox regulation (Supplemental Figure 1), we treated the rats with Dox to prevent the mutant TDP-43 transgene from further expression after the rats developed paralysis (Figure 4). To repress disease gene expression quickly, Dox was subcutaneously injected and was simultaneously given in drinking water (Figure 4A). Paralyzed rats were provided Dox-soaked soft food on the cage floor. By the fifth day of Dox treatment, mutant TDP-43 was efficiently suppressed (Figure 4A). Suppressing the mutant TDP-43 gene led to restoration of motor function in rats (Figure 4, B-D, and Supplemental Videos 1-4). Dox-treated transgenic rats began to retract hind legs by the seventh day and to totter around the cage by the ninth day of Dox treatment. Open-field assay revealed a gradual recovery of motor function in the Dox-treated rats (Figure 4E). Even 2 months after Dox treatment, grip strength recovered partially (Figure 4F). Functional recovery was dramatic but incomplete in the rats expressing mutant TDP-43 in neurons and in skeletal muscles.

Remodeling of motor units contributes to the functional recovery in Doxtreated transgenic rats. We examined the anatomical basis of functional recovery in Dox-treated transgenic rats. As expected, stereological cell counting revealed no difference in the number of motor neurons between Dox-treated and Dox-untreated ALS rats (Figure 3R). 

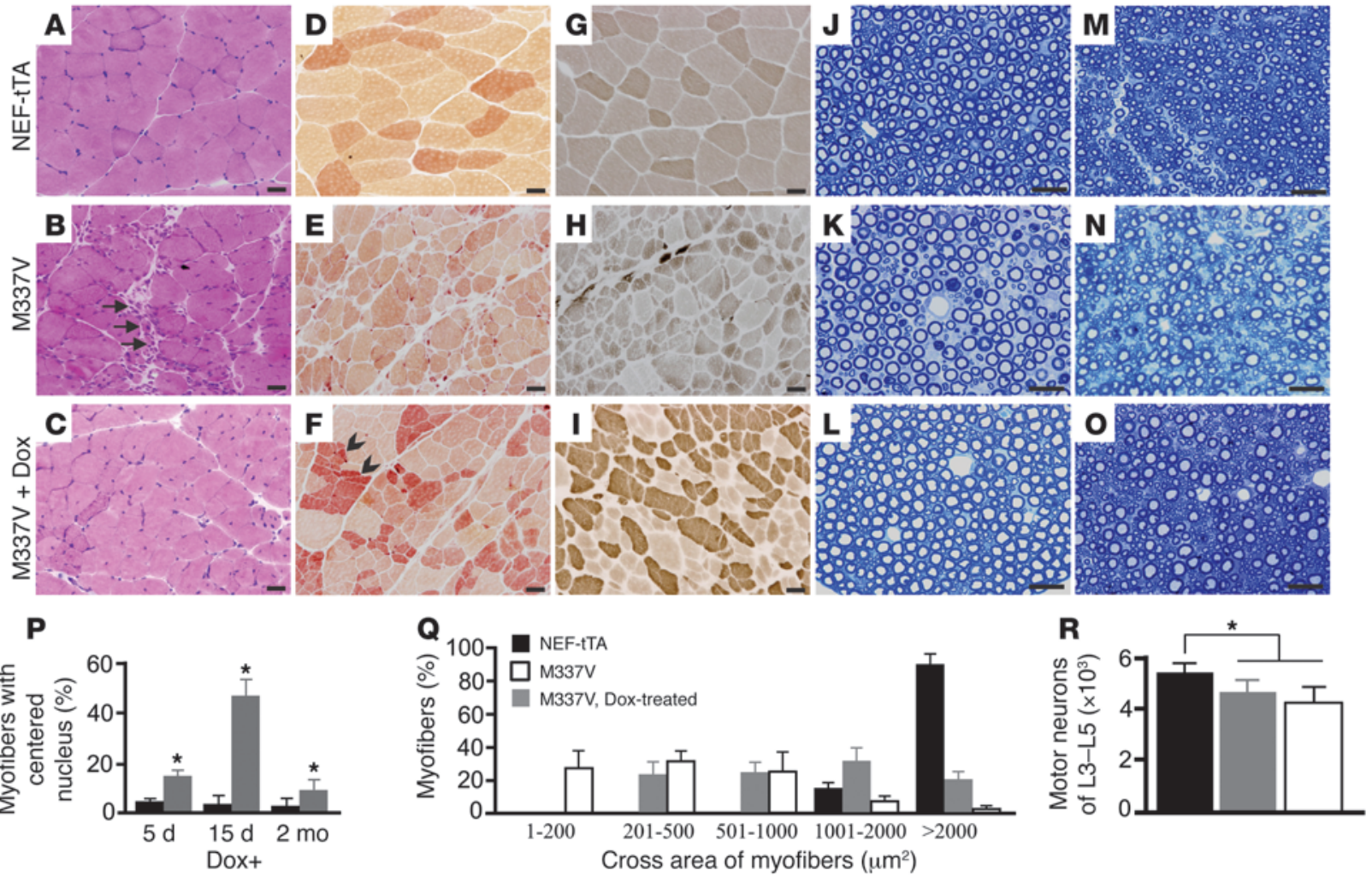

\section{Figure 3}

Motor units are remodeled in diseased rats after mutant TDP-43 is removed. (A-C) H\&E staining of gastrocnemius muscle revealed normal structure in NEF-tTA transgenic rats (A), grouped atrophy (arrows) in NEF-tTA/TRE-TDP-43 ${ }^{\mathrm{M} 337 \mathrm{~V}}$ double-transgenic rats at disease end stages (B, M337V), and regenerated muscle cells of varied sizes in diseased M337V rats after Dox treatment (C, M337V + Dox). (D-F) Histochemistry for nonspecific esterase revealed accumulated muscle fibers of varied sizes, but with similar staining (arrowheads), in M337V rats after Dox treatment. (G-I) Staining for ATPase (pH 4.6) revealed regenerated muscle cells of varied sizes in M337V rats after Dox treatment (I). (J-O) Toluidine blue staining revealed the structure of $\mathbf{L} 3$ ventral $(\mathbf{J}-\mathbf{L})$ and dorsal $(\mathbf{M}-\mathbf{O})$ roots. Scale bars: $30 \mu \mathrm{m}$. $(\mathbf{P})$ Myofibers with or without centered nucleus were quantified on the cross sections of gastrocnemius stained with $\mathrm{H} \& \mathrm{E}$. (Q) The cross areas of myofibers were quantified with ImageJ on the cross sections of gastrocnemius stained for ATPase. Quantification was done on 3 photos of the cross section of gastrocnemius, and each group contained 4 rats $(\mathbf{P}$ and $\mathbf{Q})$. Data are means $\pm \mathrm{SD}(n=4)$. $(\mathbf{R})$ Unbiased cell counting revealed the number of motor neurons $(>25 \mu \mathrm{m})$ in the lumbar cords (L3-L5). Data are means $\pm \mathrm{SD}(n=7)$. ${ }^{*} P<0.05$. All rats were deprived of Dox to induce disease at 60 days of age. Dox-untreated rats were examined at disease end stages, and Dox-treated rats were examined 2 months after Dox treatment $(\mathbf{Q}$ and $\mathbf{R})$.

Morphological analyses showed that degenerating motor axons were eliminated in Dox-treated ALS rats (Figure 3, J-Q). H\&E staining revealed that damaged muscle fibers in the gastrocnemius muscle were cleared and that muscle fibers of varied sizes were regenerated (Figure 3, A-C). Regenerating myofibers displayed centered nuclei and increased in size (Figure 3, P and Q).

While the histochemistry for nonspecific esterase and ATPase revealed normal myofibers with uniform sizes, polygonal shapes, and eccentric nuclei (Figure 3, D and G), these stainings revealed denervated myofibers with small sizes and angulated shapes (Figure 3, E and H). Nonspecific esterase staining displayed normal myofibers with pale yellow to brown color (Figure 3D), but displayed denervated or newly regenerated myofibers with red-brown color (Figure 3, E and F). ATPase staining ( $\mathrm{pH} 4$.6) can distinguish 3 types of myofibers, with light to dark colors. ATPase staining revealed a mosaic distribution of different myofibers in normal muscles (Figure 3G), but it revealed the grouping of myofibers of the same types in denervated or newly regenerated muscles (Figure 3, $\mathrm{H}$ and I). Histological analyses suggested that motor units were remodeled in Dox-treated transgenic rats.
Novel tTA transgenic rats are created to express transgenes restrictedly in motor neurons. To determine whether motor neuron death is a cell-autonomous process in TDP-43-related ALS, we developed multiple transgenic rat lines that express tTA transgene restrictedly in motor neurons (Figure 5, Supplemental Figures 4 and 5). Previous studies showed that the promoter of the choline acetyltransferase (ChAT) gene directs synthesis of transgenes selectively in spinal motor neurons in mice (32). We isolated mouse ChAT promoter from a BAC clone and used it to drive tTA transgene in rats. We obtained 3 expression lines that carry 2, 5, or 9 copies of the tTA gene (Supplemental Figure 4). Individual lines were designated as ChAT-tTA-2, -5, or -9. It is a technical challenge to measure the expression levels of transgene in a single cell population in animals. Thus, we crossed each ChAT-tTA transgenic line with TRE-TDP-43 ${ }^{\mathrm{M} 337 \mathrm{~V}}$ (line 16) rats and purified motor neurons from the double-transgenic embryos (embryonic day 14) using immunopanning (33). We then determined the expression levels of the TDP- $43^{\mathrm{M} 337 \mathrm{~V}}$ transgene in purified motor neurons and detected varying expression of human TDP-43 in individual ChAT-tTA lines (Supplemen- 
A Dox+ $0 d \quad 3 d \quad 5 d$

Tissue $\overline{\text { FB SM }} \overline{\text { FB SM }} \overline{\text { FB SM }}$
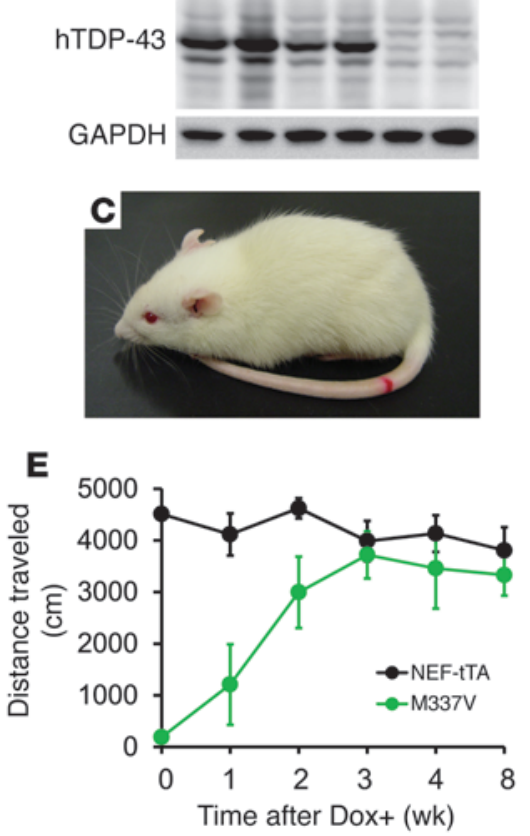
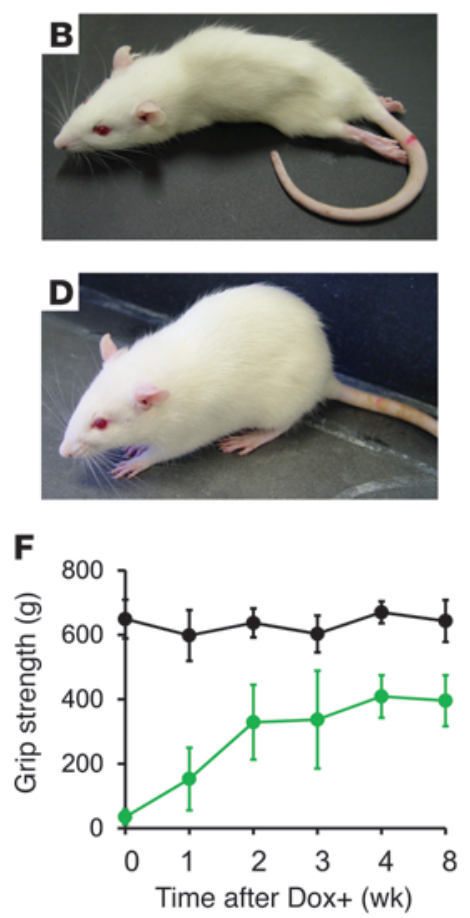

\section{Figure 4}

Suppressing mutant TDP-43 expression results in partial recovery of motor function in rats. (A) Immunoblotting revealed that Dox treatment (Dox+) suppressed the expression of mutant TDP-43 transgene (hTDP-43) as early as 5 days after Dox was administered. Equal loading was probed with an antibody to GAPDH. Tissues: FB, forebrain; SM, skeletal muscle. (B-D) Representative photographs show a M337V rat at paralysis stage (B) and at 10 days (C) and 2 months (D) after Dox treatment. (E) Open-field assay revealed a quick recovery of mobility in $\mathrm{M} 337 \mathrm{~V}$ rats after the transgene was turned off by Dox treatment (Dox+). (F) The grip strength of hind paws was partially recovered in M337V rats after the transgene was turned off. NEF-tTA denotes NEF-tTA single-transgenic rats, and M337V denotes NEF-tTA/TRE-TDP-43M337V double-transgenic rats. Data are means $\pm \operatorname{SD}(n=7)$. tal Figure 4, E-J). Compared with the NEF-tTA line, ChAT-tTA lines 5 and 9, but not 2, expressed the transgene at higher levels (Supplemental Figure 4, G, I, and J).

To facilitate determination of the transgene expression profile, we created a tTA reporter line that carries the LacZ transgene under control of the TRE promoter (Supplemental Figure 5A). We established a 3-copy TRE-LacZ line and crossed it with a ChAT-tTA-9 line to determine tTA expression profile (Figure 5, A-C, and Supplemental Figure 5, B-E). In ChAT-tTA-9/TRE-LacZ double-transgenic rats, expression of the $\mathrm{LacZ}$ gene was restricted to the ventral horn of the spinal cord (Figure 5, B and C). Doublelabel immunostaining revealed that LacZ immunoreactivity was restricted in ChAT-positive cells and that more than $60 \%$ of motor neurons expressed LacZ in the ChAT-tTA-9 line (Supplemental Figure 5, B-E). In contrast, about $40 \%$ of motor neurons expressed LacZ in the ChAT-tTA-2 and -5 lines. We then focused our studies on the ChAT-tTA-9 line.

Motor neurons undergo cell-autonomous death in TDP-43 transgenic rats. We crossed ChAT-tTA lines (ChAT-tTA-5 and ChAT-tTA9) with TDP- $43^{\mathrm{M} 337 \mathrm{~V}}$ transgenic rats (line 16 ) to examine disease induction, and we focused our studies on the ChAT-tTA-9 line, as this line expresses transgenes in the most motor neurons (Supplemental Figure 5). Similar to the reporter gene LacZ (Figure 5, B and C, and Supplemental Figure 5), mutant human TDP-43 was expressed restrictedly in the ventral horn of the spinal cord in ChAT-tTA-9/TDP-43 ${ }^{\mathrm{M} 337 \mathrm{~V}}$ rats (Figure 5, D and E). Double-label immunostaining revealed that human TDP-43 was colocalized with the motor neuron marker ChAT (Figure 5, J-L), but was not colocalized with the oligodendrocyte marker APC (Figure 5F) or the astrocyte marker GFAP (Figure 5, G-I). The results suggest that expression of TDP- $43^{\mathrm{M} 337 \mathrm{~V}}$ is restricted in spinal motor neurons.

To induce disease phenotypes in adult rats, we gave transgenic rats Dox in drinking water until the rats were 60 days old (Figure 6A and Supplemental Figure 6A). Sprague Dawley rats are sexually mature at the age of 70 days. In ChAT-tTA-9/TDP-43 ${ }^{\mathrm{M} 337 \mathrm{~V}}$ transgenic rats, human TDP-43 was undetectable in the presence of Dox, but was detected by 3 days off Dox (Figure 6B). Human TDP-43 was not detectable in the skeletal muscles (Figure 6B). Soon after expression of mutant TDP-43, rats began losing grip strength and motor activity, as revealed by open-field assay and grip-strength measure (Figure 6, C and D). By the age of 70 days (10 days off Dox), most mutant rats developed the early signs of paralysis (Figure 6E). Rats reached disease end stages within a week after disease onset (Figure 6F). On average, the duration of disease progression was less than 2 weeks (Figure 6, E and F). Unbiased stereological counting revealed that more than $60 \%$ of spinal motor neurons were lost in mutant TDP-43 transgenic rats at disease end stages (Figure 6, G-K). Interestingly, the size of surviving motor neurons and the volume of ventral lumbar cords were unaltered at disease end stages (Figure 6, L and M). Toluidine blue staining revealed that axons in the ventral roots (motor axons), but not in the dorsal roots (sensory axons), were damaged (Figure 7, N-S). As a result of motor neuron death, groups of skeletal muscle fibers were atrophied, as revealed by H\&E staining (Figure 7, E and F) and by histochemistry for nonspecific esterase (Figure 7, $\mathrm{H}$ and I) and ATPase (Figure 7, K and L). Restricted overexpression of mutant TDP-43 in motor neurons caused a severe loss of motor neurons and motor axons and caused a denervation atrophy of skeletal muscles in ChAT-tTA-9/TDP-43 $3^{\mathrm{M} 37 \mathrm{~V}}$ transgenic rats.

We next characterized ChAT-tTA-5/TDP-43 ${ }^{\mathrm{M} 337 \mathrm{~V}}$ transgenic rats (Supplemental Figure 6). When Dox was withdrawn at the age of 60 days, rats displayed disease onset by the age of 85 days (Supplemental Figure 6, A and B), but did not reach disease end stages by the age of 150 days. The ChAT-tTA- 5 line directed transgene expression in less than $50 \%$ of spinal motor neurons (Supplemental Figure 5E), and thus, ChAT-tTA-5/TDP-43 ${ }^{\mathrm{M} 337 \mathrm{~V}}$ rats would not lose enough motor neurons to cause full paralysis. Indeed, only about $30 \%$ of motor neurons were lost in the rats by 150 days of 

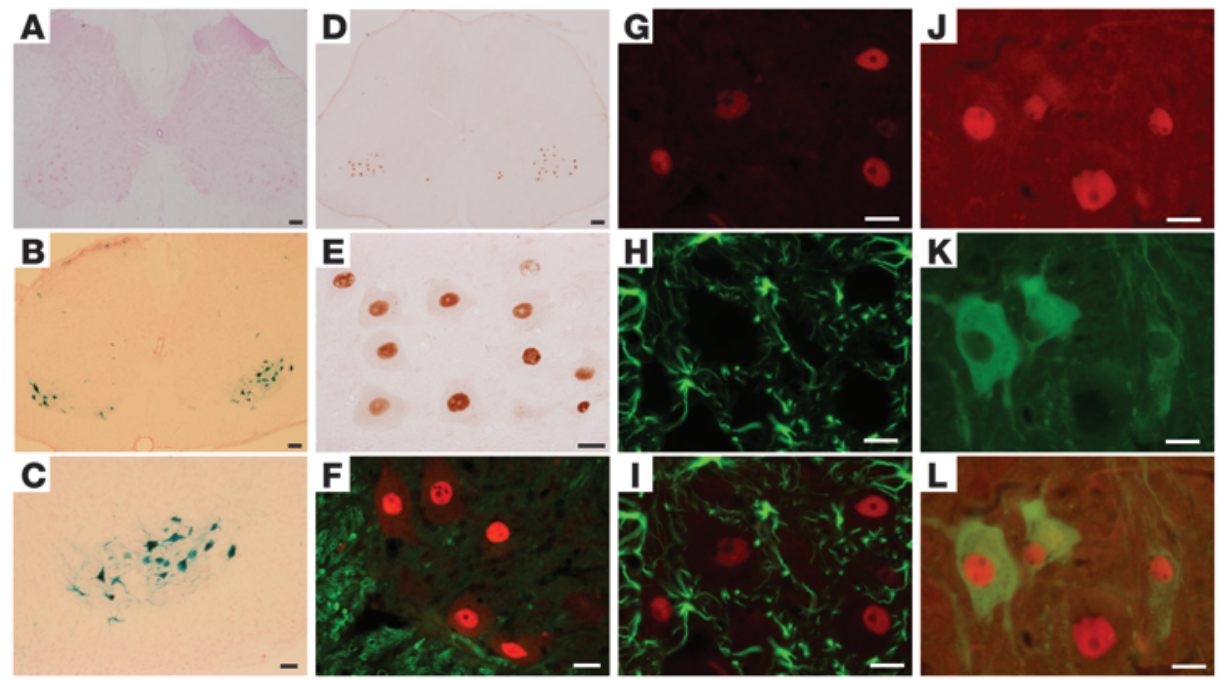

\section{Figure 5}

Restricted overexpression of mutant TDP-43 in motor neurons is achieved in transgenic rats. (A-C) $X$-gal staining revealed that the positive cells were restricted to the ventral horns of the lumbar cord in ChAT-tTA-9/TRE-LacZ double-transgenic (B and $\mathbf{C}$ ), but not in TRE-LacZ single-transgenic (A), rats. Cross sections of lumbar cords were first stained with $X$-gal and then were counterstained with fast red. (D and E) Immunohistochemistry revealed that human TDP-43 immunoreactivity was restricted in large cells in the ventral horns of the lumbar cord. ChAT-tTA-9/TRE-TDP-43M337V transgenic rat at 70 days old was examined for human TDP-43 expression at 10 days after Dox withdrawal. (F-L) Double-label immunofluorescence staining revealed that human TDP-43 (red, F, G, I, J, and L) was not colocalized with APC (a marker of oligodendrocytes; F, green) or GFAP (a marker of astrocytes; $\mathbf{H}$ and $\mathbf{I}$, green), but was colocalized with ChAT (a marker of motor neurons; $\mathbf{K}$ and $\mathbf{L}$, green). Scale bars: $100 \mu \mathrm{m}$ (A, B, and D); $50 \mu \mathrm{m}(\mathbf{C}) ; 20 \mu \mathrm{m}(\mathbf{E}-\mathbf{L})$.

age (Supplemental Figure 6C). Accordingly, fewer motor axons in the ventral roots were lost in ChAT-tTA-5/TDP- $43^{\mathrm{M} 337 \mathrm{~V}}$ rats compared with ChAT-tTA-9/TDP-43 ${ }^{\mathrm{M} 337 \mathrm{~V}}$ rats (Supplemental Figure 6, $\mathrm{H}$ and I, and Figure 7, N-P). Overexpression of mutant TDP-43 at a low level and in fewer motor neurons caused moderate paralysis phenotypes in rats (Supplemental Figure 6).

Removal of mutant TDP-43 prevents disease from progression in rats expressing the disease gene restrictedly in motor neurons. Compared with NEF-tTA/TDP-43 ${ }^{\mathrm{M} 337 \mathrm{~V}}$ rats (Figure 2), ChAT-tTA-9/ TDP- $43^{\mathrm{M} 337 \mathrm{~V}}$ rats displayed a more severe loss of motor neurons, but displayed a similar progression of paralysis phenotype (Figure 6). We determined whether disease progression could be halted by stopping mutant TDP-43 expression after disease onset in ChAT-tTA-9/TDP-43 ${ }^{\mathrm{M} 337 \mathrm{~V}}$ rats. When Dox was withdrawn from ChAT-tTA-9/TDP-43 ${ }^{\mathrm{M} 337 \mathrm{~V}}$ rats at the age of 60 days (Figure $6 \mathrm{~A}$ ), the rats developed an early paralysis phenotype by 70 days of age and reached disease end stages by 78 days of age (Figure 6, C-F). Dox was thus withdrawn from rats that were 60 days old and was added back 12 days later, so that mutant TDP-43 was expressed in the rats from 63 to 77 days of age (Figure 6B and Figure 7B). We refer to restoring Dox as a treatment. Dox-untreated and Dox-treated rats all developed paralysis at comparable speeds before Dox produced an effect on disease gene expression (Figure 7C). Soft food was provided to rats with paralysis. While the disease rapidly progressed to end stages in Dox-untreated rats, the disease stopped progressing at paralysis stages in Doxtreated rats (Figure 7, C and D). Within a few days, Dox-treated rats began to gain body weight and to gain partial recovery of motor activity (Figure 7, C and D). Dox-untreated rats were ter- microgliadiffersin the spinal cord of ALS rats. Glial cells play an important role in motor neuron degeneration (34-36). We examined glial reaction to motor neuron death in ChAT-tTA-9/TDP-43 ${ }^{\mathrm{M} 337 \mathrm{~V}}$ transgenic rats. Astrocytes and microglia were activated in the spinal cords of paralyzed rats (Figure 8). Mutant TDP-43 transgenic rats lost a large quantity of motor neurons at disease end stages (Figure 6G). In rats with active disease, reactive astrocytes distributed across the whole spinal cord and were not restricted to the ventral horns, where motor neurons expressing mutant TDP-43 were dying (Figure 8, B, E, and N), but microglia were activated around diseased motor neurons in the ventral horns (Figures 8, H and K). NG2-positive glia were also activated around diseased motor neurons (Figure 8, P-R). When disease progression was halted by Dox treatment (Figure 7, A-D), microglial reaction was quenched (Figure 8 , I and $\mathrm{L}$ ) and reactive astrocytes were restricted to the ventral horns where damaged motor neurons resided (Figure 8, C, F, and O). Varied response of glial cells may suggest a different role for astrocytes and microglia in the propagation and repair of neuronal damage in the disease.

Ubiquitin accumulates in motor neurons expressing mutant TDP-43 in rats with paralysis. Similar to NEF-tTA/TDP- $43^{\mathrm{M} 337 \mathrm{~V}}$ rats, ChATtTA-9/TDP-43 ${ }^{\mathrm{M} 337 \mathrm{~V}}$ rats did not develop TDP-43 inclusion in diseased motor neurons (Supplemental Figure 7). As the disease progressed, mutant TDP-43 accumulated in the cytoplasm of motor neurons in transgenic rats (Supplemental Figure 7). In NEF-tTA/ $\mathrm{TDP}-43^{\mathrm{M} 337 \mathrm{~V}}$ rats at disease end stages, rare ubiquitin aggregates were formed only in cortical neurons (Supplemental Figure 3). In contrast, ubiquitin accumulated in diseased motor neurons in ChAT-tTA-9/TDP-43 ${ }^{\mathrm{M} 337 \mathrm{~V}}$ rats (Figure 9 and Supplemental Figure 8). Accumulated ubiquitin delineated the cell body and neurites 
A
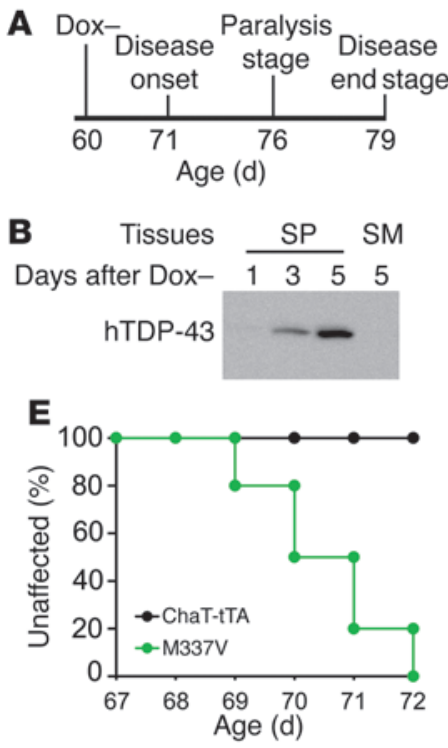

C
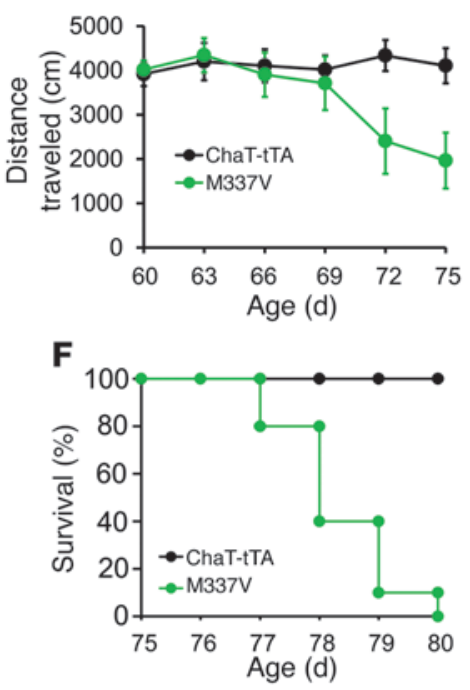

D
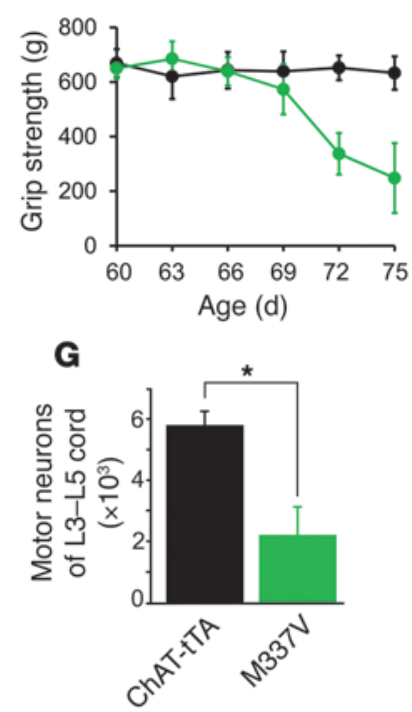
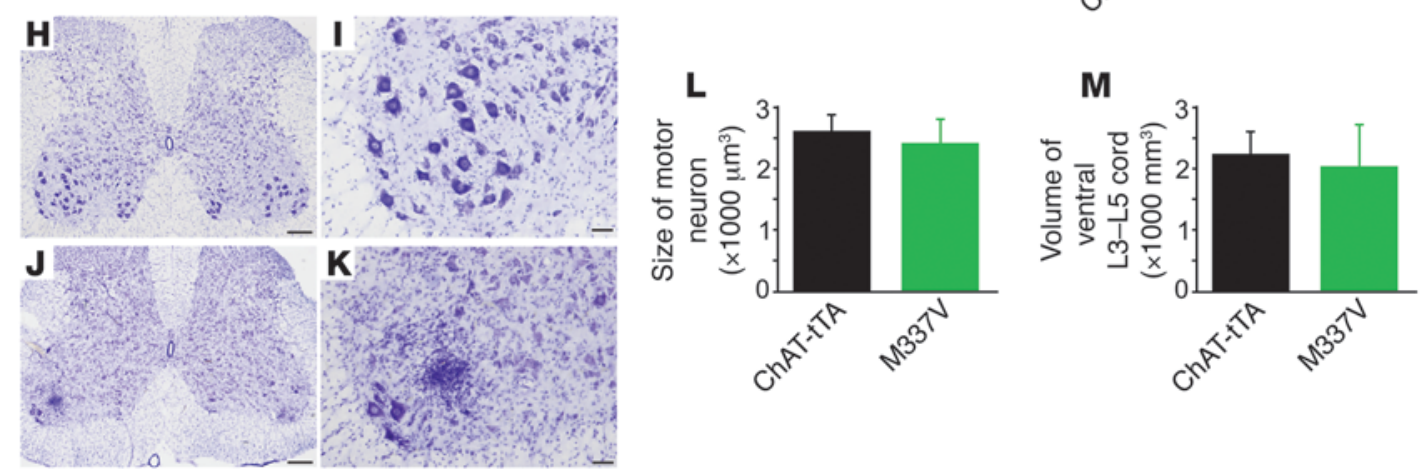

\section{Figure 6}

Restricted overexpression of mutant TDP-43 in motor neurons results in progressive paralysis and substantial motor neuron death. (A) A diagram shows the course of gene induction and disease progression. ChAT-tTA-9/TRE-TDP-43M337V double-transgenic (M337V) rats were deprived of Dox (Dox-) at 60 days of age. (B) Immunoblotting revealed induction of mutant TDP-43 transgene (hTDP-43) after Dox withdrawal (Dox-). (C) Open-field assay measured mobility within 20 minutes. ChAT-tTA denotes ChAT-tTA-9 (line 9) transgenic rats. (D) The grip strength of 2 hind paws was measured daily. Data are means $\pm \mathrm{SD}(\mathbf{C}$ and $\mathbf{D}, n=10)$. (E and $\mathbf{F})$ Graphs show the probability of disease onset and rat mortality. Rats were counted at death when they reached disease end stages. 10 rats of equal sex composition were assessed in $\mathbf{E}$ and $\mathbf{F}$. (G) Stereological cell counting revealed a profound loss of motor neurons (>25 $\mu \mathrm{m}$ in diameter) in the L3-L5 cords of M337V rats at end stages as compared with ChAT-tTA rats at matched ages. Data are means \pm SD $(n=8) .{ }^{*} P<0.01$. ( $\left.\mathbf{H}-\mathbf{K}\right)$ Representative photos of low $(\mathbf{H}$ and $\mathbf{J})$ and high $(\mathbf{I}$ and K) magnification show the lumbar cord of a M337V rat at disease end stage ( $\mathbf{J}$ and $\mathbf{K}$ ) or a ChAT-tTA rat at matched age (H and I). Scale bars: $200 \mu \mathrm{m}(\mathbf{H}$ and $\mathbf{J}) ; 60 \mu \mathrm{m}(\mathbf{I}$ and $\mathbf{K})$. ( $\mathbf{L}$ and $\mathbf{M})$ Graphs show the average size of motor neurons in L3-L5 cords and the total volume of the ventral horns of L3-L5 cord. Data are means \pm SD $(n=8)$.

of diseased motor neurons expressing mutant TDP-43 (Figure 9E and Supplemental Figure 8, B and C). Motor neurons with ubiquitin aggregates were few at disease onset, but were remarkably increased at paralysis stages (Supplemental Figure 8G). Accumulated ubiquitin was detectable only in rats with active disease (Figure 9, B, E, and G-I), but not in rats with disease halted by Dox treatment (Figure 9, C and F). The results suggest that accumulated ubiquitin may be cleared after the disease is halted and that ubiquitin accumulation may be an accompanying event of motor neuron death in the disease caused by TDP-43 mutation.

\section{Discussion}

TDP-43 proteinopathy is implicated in sporadic ALS and FTLD $(2,10)$. Mutation of TDP-43 causes an inherited form of ALS (1, 37-39). Most TDP-43 transgenic animal models reported on develop a profound loss of cortical neurons, but only a few lines develop a limited loss of motor neurons at disease end stages $(3-9,40)$. To induce motor neuron death, mutant TDP-43 may not be required to present in all types of related cells. To address these questions, we created transgenic rats expressing mutant human TDP-43 in inducible, reversible, and cell-specific manners. Mutant TDP-43 in motor neurons alone was sufficient to induce motor neuron degeneration. Even at paralysis stages, stopping the disease gene from further expression prevented paralysis from progression and resulted in dramatic recovery of motor function in mutant TDP-43 transgenic rats.

NEF-tTA/TDP-43 $3^{\text {M337V }}$ and ChAT-tTA/TDP-43 $3^{\text {M337V }}$ transgenic rats developed a similar disease phenotype - progressive paralysis - reminiscent of ALS that is characterized by progressive degeneration of motor neurons and denervation atrophy of 
A

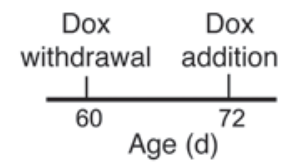

\begin{tabular}{lll} 
B & \multicolumn{2}{c}{ SP } \\
\cline { 2 - 4 } & 0 & $2 \quad 4 \quad 6$
\end{tabular}

hTDP-43
C

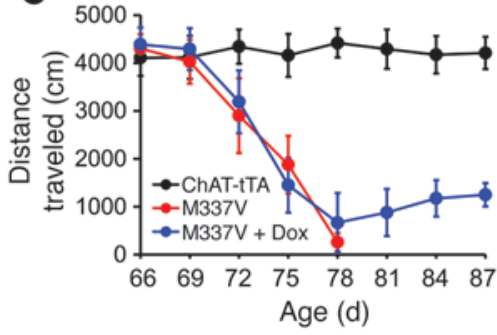

D

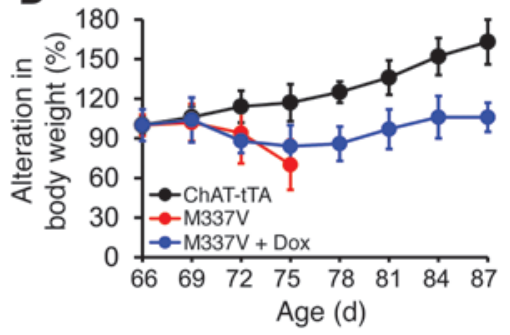

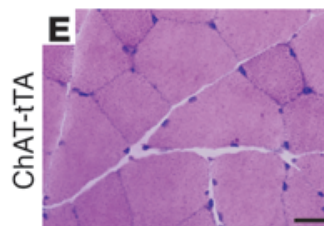
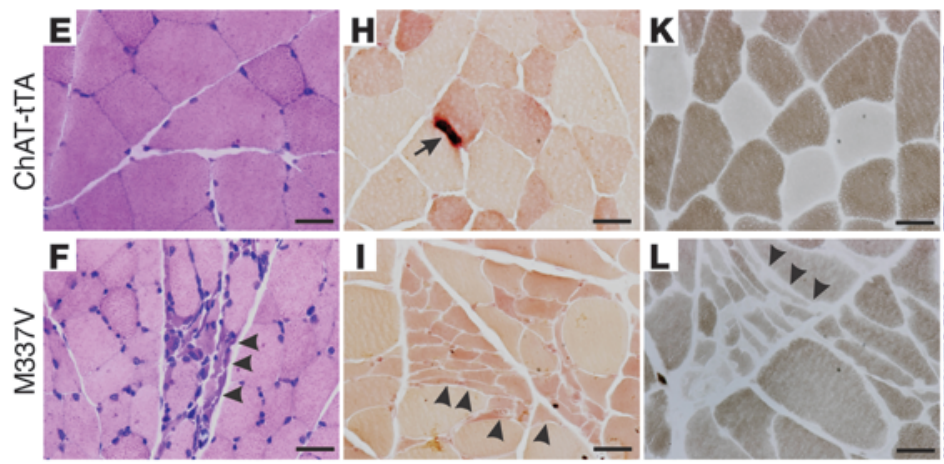

M
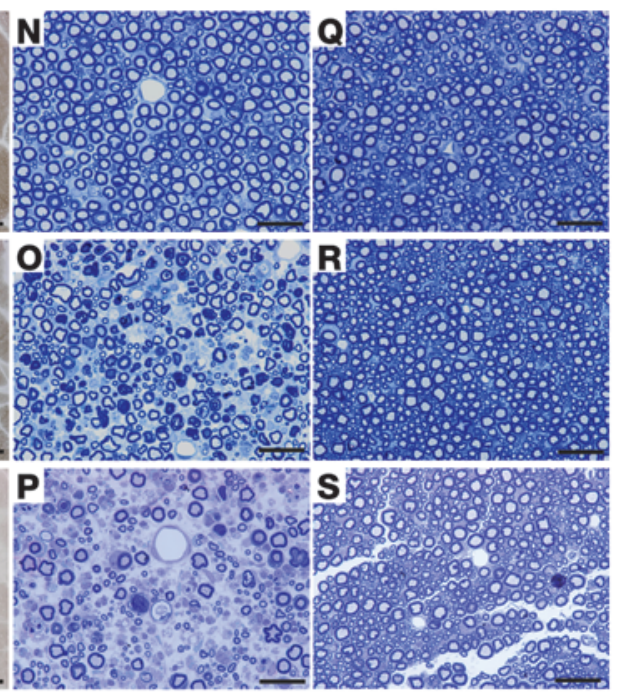

V

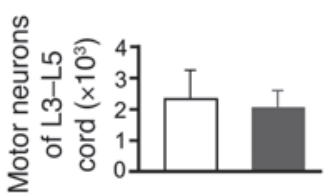

\section{Figure 7}

Suppressing mutant TDP-43 expression prevents disease from progression in rats. (A) A diagram shows induction (Dox withdrawal) and suppression (Dox addition) of mutant TDP-43 (hTDP-43) transgene. (B) Immunoblotting revealed gene suppression after Dox addition (Dox+). (C) Open-field assay measured mobility within 20 minutes. ChAT-tTA denotes ChAT-tTA-9 rats, and M337V denotes ChAT-tTA-9/TRE-TDP-43M337V rats. (D) The body weight of an individual rat at 66 days of age was used as the base for calculating alteration in body weight. Data are means \pm SD ( $\mathbf{C}$ and $\mathbf{D}, n=8)$. (E-M) H\&E staining $(\mathbf{E}-\mathbf{G})$ and histochemistry for nonspecific esterase $(\mathbf{H}-\mathbf{J})$ and ATPase $(\mathbf{K}-\mathbf{M})$ revealed grouped atrophy of skeletal muscle in Dox-untreated $(\mathbf{F}, \mathbf{I}$, and $\mathbf{L})$ and varied sizes of muscle fibers in Dox-treated $(\mathbf{G}, \mathbf{J}$, and $\mathbf{M})$ rats. Arrowheads point to grouped atrophy, and arrow points to a neuromuscular junction. (N-S) Toluidine blue staining showed L3 ventral (N-P) and dorsal (Q-S) roots. All scale bars: $30 \mu \mathrm{m}$. (T) Myofibers were quantified on cross sections of gastrocnemius stained with H\&E. (U) The cross areas of myofibers were quantified with ImageJ on cross sections of gastrocnemius stained for ATPase. Quantification was done on 3 photos of the cross sections (T and $\mathbf{U}$ ). Data are means \pm SD ( $n=4$ rats). ${ }^{*} P<0.05$. (V) The number of motor neurons $(>25 \mu \mathrm{m})$ is not different between Dox-treated and -untreated M337V rats. The group of Dox-untreated M337V rats is identical to the M337V group of Figure 6G. Dox-treated rats were terminated at 90 days of age. Data are means $\pm \operatorname{SD}(n=8)$.

skeletal muscles. The disease progressed rapidly to end stages in these rat models. At disease end stages, NEF-tTA/TDP-43 $4337 \mathrm{~V}$ transgenic rats suffered from severe damage to motor units that included impaired motor axons and grouped atrophy of skeletal muscles, although a moderate loss of spinal motor neurons was observed. In contrast, ChAT-tTA/TDP-43 ${ }^{\mathrm{M} 337 \mathrm{~V}}$ rats suffered a heavy loss of motor neurons at disease end stages. Motor function depends on the integrity of motor circuitry, including motor neurons, motor axons, neuromuscular junctions, and skeletal muscles. Damage to any of these elements may promote onset and progression of paralysis. Both NEF-tTA/TDP-43 ${ }^{\mathrm{M} 337 \mathrm{~V}}$ and ChAT-tTA/TDP- $43^{\mathrm{M} 337 \mathrm{~V}}$ transgenic rats reproduced the core phenotypes of ALS.

ChAT-tTA/TDP-43 ${ }^{\mathrm{M} 337 \mathrm{~V}}$ rats expressed mutant TDP-43 restrictedly in spinal motor neurons and developed a severe degeneration of motor neurons. The results suggest that mutant TDP-43 in motor neurons alone is sufficient to kill the cells. In response to TDP-43 mutation, motor neurons can undergo cell-autonomous death. Our findings in TDP-43 transgenic rats strikingly contrasts with the findings in SOD1 transgenic mice that do 

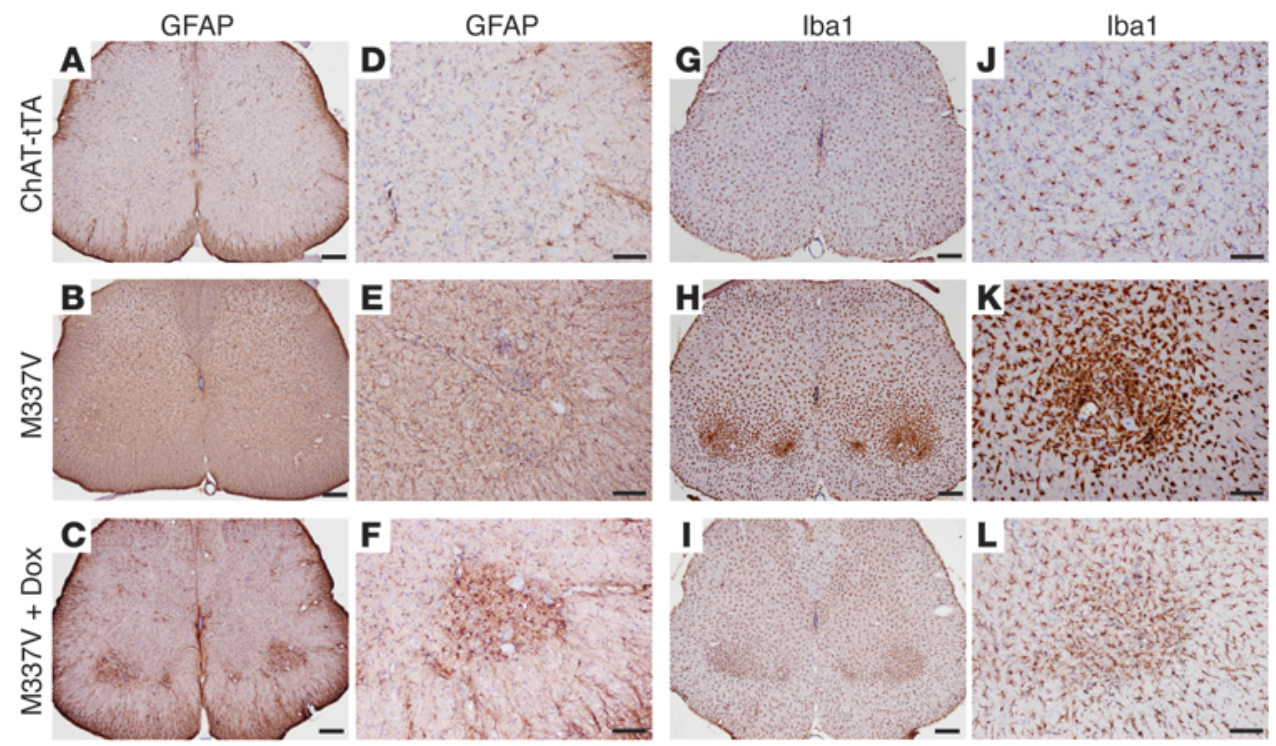

ChAT-tTA
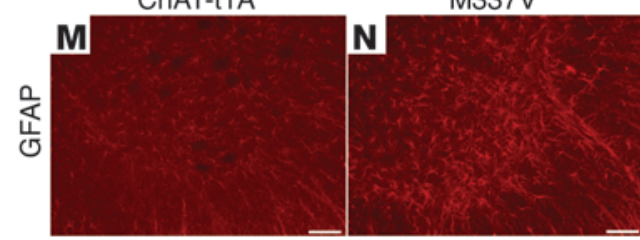

M337V
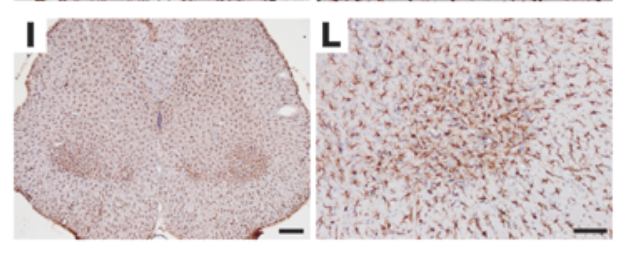

M337V + Dox

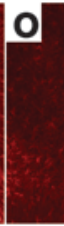

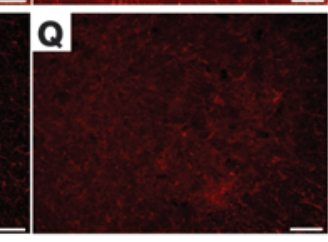
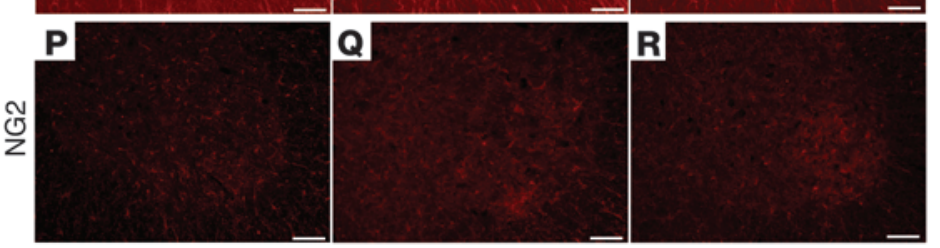

\section{Figure 8}

Astrocytes and microglia react to motor neuron death in rats. (A-L) Immunohistochemistry revealed marked activation of astrocytes (GFAP) and microglia (Iba1) in the spinal cords of mutant TDP-43 transgenic rats. (M-R) Immunofluorescence staining showed the distribution of astrocytes (GFAP) and NG2 glia in the ventral horns of lumbar cords. ChAT-tTA-9/TRE-TDP-43M337V double-transgenic (M337V) rats were deprived of Dox at 60 days of age and were completely paralyzed (end stage) by 78 days of age. ChAT-tTA-9 single-transgenic rats were terminated at matched ages. Randomly chosen M337V rats were treated with Dox (M337V + Dox) to prevent mutant TDP-43 from further expression after disease onset. Dox-treated rats were terminated at 90 days of age. Scale bars: $200 \mu \mathrm{m}(\mathbf{A}-\mathbf{C}$ and $\mathbf{G}-\mathbf{I}) ; 100 \mu \mathrm{m}$ (D-F, J-L, and M-R). not develop motor neuron degeneration when mutant SOD1 is overexpressed restrictedly in motor neurons $(25,41)$, underscoring the difference in pathogenesis between TDP-43 and SOD1 mutations. Our findings do not necessarily exclude that cells other than motor neurons play an important role in TDP-43 pathogenesis. In fact, the severity of disease is not correlated with the expression levels of mutant TDP-43 in motor neurons in our transgenic rats. While ChAT-tTA/TDP-43 ${ }^{\mathrm{M} 337 \mathrm{~V}}$ rats express mutant TDP-43 restrictedly in motor neurons, NEF-tTA/TDP-43 ${ }^{\mathrm{M} 337 \mathrm{~V}}$ rats express the gene in motor neurons and in other cells, including the other neurons and skeletal muscles. Compared with ChAT-tTA-9/TDP-43 ${ }^{\mathrm{M} 337 \mathrm{~V}}$ rats, NEF-tTA/TDP-43 ${ }^{\mathrm{M} 337 \mathrm{~V}}$ rats expressed less mutant TDP-43 protein in motor neurons and developed paralysis at comparable speeds, implying that cells other than motor neurons may also play an important role in TDP-43 pathogenesis. It remains to be determined whether restricted expression of mutant TDP-43 in the other cells, such as astrocytes and glia, is sufficient to induce motor neuron degeneration. Mutant TDP-43 in motor neurons alone is sufficient to promote onset and progression of neurodegeneration. Although overexpression of a mutant form of TDP-43 in motor neurons is toxic in rats, this may not be representative of the consequences of this mutation in patients as (a) it is not known whether this mutation causes this extent of overexpression of TDP-43 in patients, (b) the disease course is much more rapid in rats than in patients, implying that the underlying mechanisms may be different, and (c) patients do not express mutant TDP-43 specifically in motor neurons. Therefore, ultimately, direct relevance to the disease in humans is not known and remains to be tested.

Our 2 individual ChAT-tTA lines expressed mutant TDP-43 restrictedly in motor neurons and at varying levels. ChAT-tTA-5/ TDP-43 $3^{\mathrm{M} 37 \mathrm{~V}}$ and ChAT-tTA-9/TDP-43 ${ }^{\mathrm{M} 337 \mathrm{~V}}$ transgenic rats developed paralysis at similar ages, but ChAT-tTA-5/TDP- $43^{\mathrm{M} 337 \mathrm{~V}}$ rats displayed a much slower progression of the disease because this line expressed less mutant TDP-43 protein in fewer motor neurons. In ALS patients, motor symptoms often begin focally and progress contiguously. Consistently with focal onset of motor symptoms, loss of lower motor neurons first occurs in the corresponding region of brain stem or spinal cord and propagates to the other regions $(42,43)$. At postmortem analysis, more than $60 \%$ of total lower motor neurons are lost and the neuronal loss is more severe in the region first affected $(42,43)$. Most ALS patients die within 3 years, but some survive more than 10 years. Our 2 individual transgenic lines reproduce the variation of disease progression in ALS, albeit with a time frame of weeks rather than years. Mutations of TDP-43 and SOD1 are all linked to familial ALS. While WT SOD1 is an excellent control for mutation in the gene in transgenic studies, WT TDP-43 may not be. While pathogenic mutation of TDP-43 definitely causes an inherited form of ALS, TDP-43 is found to be ubiquitinated, hyperphosphorylated, and aggregated in sporadic ALS, implying that WT TDP-43 may play a role in ALS pathogenesis. Overexpression of the mutant, but not 


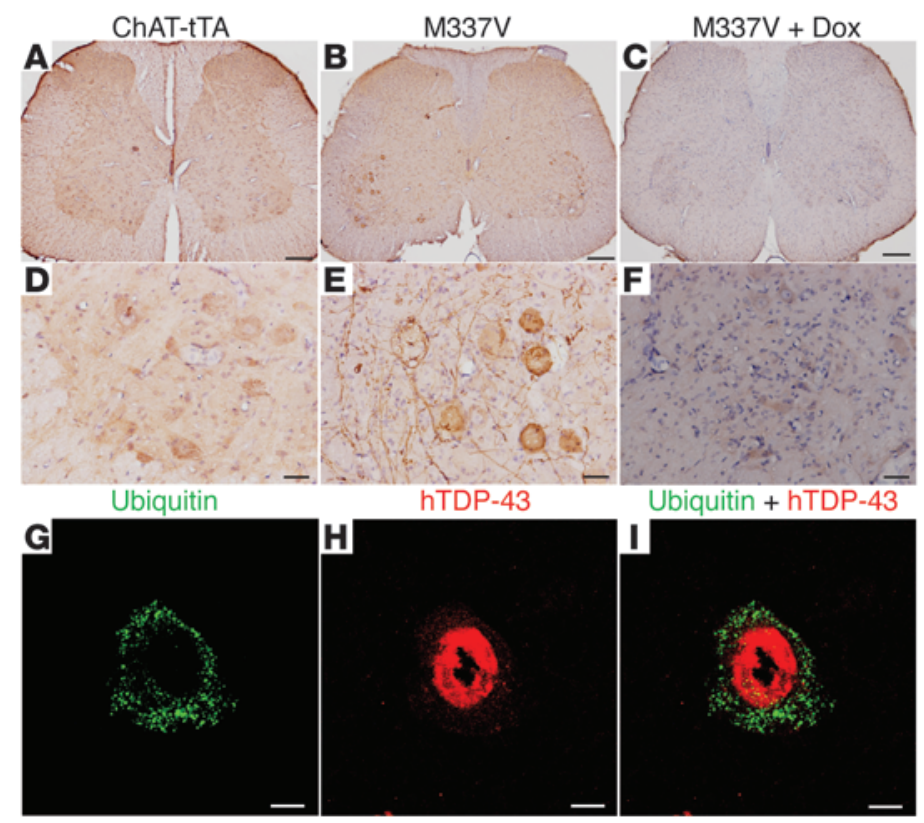

Figure 9

Ubiquitin accumulates in the motor neurons of ALS rats. (A-F) Immunostaining revealed a marked accumulation of ubiquitin in the motor neurons of mutant TDP-43 transgenic rats. (G-I) Confocal microscopy revealed that ubiquitin aggregates accumulated in the motor neurons expressing mutant human TDP-43 (hTDP-43). ChAT-tTA-9/TRE-TDP-43M337V double-transgenic (M337V) rats were deprived of Dox at 60 days of age and were paralyzed at the age of 78 days. ChAT-tTA-9 single-transgenic (ChAT-tTA) rats were terminated at matched ages. Some M337V rats were treated with Dox (M337V + Dox) to prevent mutant TDP-43 from further expression after disease onset. Dox-treated rats were terminated at 90 days of age. Scale bars: $200 \mu \mathrm{m}$ (A-C); $50 \mu \mathrm{m}$ (D-F); $10 \mu \mathrm{m}$ (G-I).

the normal, SOD1 causes ALS phenotypes in rodents $(27,44,45)$. In striking contrast, overexpression of both the mutant and the normal TDP-43 causes neurodegeneration and disease phenotypes in rodents, although mutant TDP-43 appears to be more toxic than the WT form $(3,7)$. Previous findings suggest that elevated levels of normal protein TDP-43 are sufficient to cause the disease. Indeed, a genetic variant in the $3^{\prime}$ UTR region of TDP-43 causes a significant increase in TDP-43 expression, and this TDP-43 variant has been associated with ALS and FTLD in 2 unrelated families (46). Further, pathogenic mutation in TDP-43 elongates the lifetime of TDP-43 protein and thus possibly increases the levels of TDP-43 protein (47). While the functional effect of the TDP-43 mutation on the protein remains to be determined, pathogenic mutation and elevated expression of TDP-43 prove neurotoxic. Here, we used mutant TDP-43 transgenic rats as one model for testing the reversibility of motor neuron degeneration.

Disease progression in both NEF-tTA/TDP-43 $3^{\mathrm{M} 33 \mathrm{~V}}$ and ChATtTA-9/TDP-43 ${ }^{\mathrm{M} 337 \mathrm{~V}}$ rats was halted after expression of mutant TDP-43 was suppressed by Dox. Rats of these 2 lines began expressing mutant TDP-43 by 5 days off Dox. The speed of disease progression and the duration of disease were comparable between NEF-tTA/TDP-43 ${ }^{\mathrm{M} 337 \mathrm{~V}}$ and ChAT-tTA-9/TDP-43M337V rats. Mutant TDP-43 transgene was turned on in NEF-tTA/ TDP-43 ${ }^{\mathrm{M} 337 \mathrm{~V}}$ and ChAT-tTA-9/TDP-43 ${ }^{\mathrm{M} 337 \mathrm{~V}}$ rats at the age of 60 days, so that rats of these 2 lines reached disease end stages at similar ages. Dox was added back to NEF-tTA/TDP-43 ${ }^{\mathrm{M} 337 \mathrm{~V}}$ rats at paralysis stages and to ChAT-tTA-9/TDP-43 $3^{\mathrm{M} 37 \mathrm{~V}}$ rats at disease onset. Mutant TDP-43 expression was stopped at 5 days on Dox in NEF-tTA/TDP-43 ${ }^{\mathrm{M} 337 \mathrm{~V}}$ rats and at 6 days on Dox in ChAT-tTA-9/TDP-43 ${ }^{\mathrm{M} 337 \mathrm{~V}}$ rats. By the time of mutant TDP-43 suppression, NEF-tTA/TDP-43 ${ }^{\mathrm{M} 337 \mathrm{~V}}$ and ChAT-tTA-9/TDP-43 ${ }^{\mathrm{M} 337 \mathrm{~V}}$ rats approached disease end stages. Soon after the effect of Dox, progression of ALS halted in Dox-treated rats. Dox treatment resulted in a dramatic recovery of motor function in NEF-tTA/TDP-43 ${ }^{\mathrm{M} 337 \mathrm{~V}}$ rats, but only resulted in a moderate recovery of motor function in ChAT-tTA-9/TDP-43 ${ }^{\mathrm{M} 337 \mathrm{~V}}$ rats. By the effectiveness of Dox, NEF-tTA/TDP-43 ${ }^{\mathrm{M} 337 \mathrm{~V}}$ rats lost about $20 \%$ of spinal motor neurons and ChAT-tTA-9/TDP-43 $3^{\mathrm{M} 337 \mathrm{~V}}$ rats lost more than $60 \%$ of the neurons, although denervation atrophy of skeletal muscles was remarkable in all these rats. The potential of functional recovery in TDP-43 transgenic rats largely depends on the viability of motor neurons. Compared with RNAi-mediated gene silencing (18-20), the Tet regulatory system turns on and off regulated genes readily and efficiently and is convenient for determining the reversibility of disease phenotypes in animal models (29). Our findings suggest that motor neuron degeneration can be stopped and the disease can be prevented from progression, provided that an effective treatment is developed.

In addition, TDP-43 inclusion was not detected in NEF-tTA/ TDP-43 ${ }^{\mathrm{M} 337 \mathrm{~V}}$ and ChAT-tTA/TDP-43 ${ }^{\mathrm{M} 337 \mathrm{~V}}$ rats. Consistent with previous findings in TDP-43 transgenic rats and mice $(3,7)$, TDP-43 inclusion is rare and is not essential to neuron death. In the absence of TDP-43 inclusion, TDP-43 accumulated in the cytoplasm as disease progressed. Few neurons in the cortex of NEF-tTA/TDP-43 $3^{\mathrm{M} 337 \mathrm{~V}}$ rats contained clear ubiquitin aggregates. Diseased motor neurons were full of accumulated ubiquitin in ChAT-tTA/TDP-43 $3^{\mathrm{M} 33 \mathrm{~V}}$ rats as disease progressed. Intriguingly, ubiquitin aggregates were not detectable after the disease stopped progressing. Gliosis was remarkable and was related to active motor neuron degeneration in TDP-43 transgenic rats. Although how motor neurons die in ALS remains to be determined, ubiquitin aggregation, gliosis, and cytoplasmic accumulation of TDP-43 are correlated to motor neuron death in mutant TDP-43 transgenic rats. In summary, we observed that motor neuron degeneration is partially reversible, at least in TDP-43 transgenic rats.

\section{Methods}

Generation of transgenic rats. Transgenic rats were created on a sole Sprague Dawley background as described previously (29). The promoters of the human NEF gene and the mouse ChAT gene were isolated from BAC clones (CHORI: RP11-91J21 and RP24-256F2). Selected NEF and ChAT promoters drove the $t T A$ gene. The resulting constructs NEF-tTA and ChAT-tTA were linearized and injected into the pronuclei of rat single-cell embryos to produce transgenic founders. Transgenic rats were identified by PCR analysis of rat tail DNA with the following primers: NEF-tTA, 5'-TCCACTTTGAGGGGTCTCTG-3' (forward) and 5'-AGCATCTCATCACTTCCCTG-3' (reverse); and ChAT-tTA, 5'-TCCAAGGCAGAGTTGATGAC-3' (forward) and $5^{\prime}$-TGAGTTCCAGGCAAACCAAG-3' (reverse). LacZ transgene was assembled downstream of the TRE promoter, and TRE-LacZ transgenic rats were created (29). TRE-LacZ transgenic rats were identified by PCR analysis with the following primers: 5'-TGACCTCCATAGAAGACACC-3' (forward) and 5'-TCACTGCATTCTAGTTGTGGT-3' (reverse). 
Dox treatment. To suppress expression of the TDP- $43^{\mathrm{M} 337 \mathrm{~V}}$ transgene in NEF-tTA/TDP-43 $3^{\mathrm{M} 337 \mathrm{~V}}$ and ChAT-tTA/TDP-43 ${ }^{\mathrm{M} 337 \mathrm{~V}}$ rats in embryonic and postnatal stages, Dox was constantly given to breeding rats in drinking water $(50 \mu \mathrm{g} / \mathrm{ml})$ as described previously $(3,31)$. To restore TDP-43 $3^{\mathrm{M} 337 \mathrm{~V}}$ expression, Dox was withdrawn from NEF-tTA/TDP-43 ${ }^{\mathrm{M} 337 \mathrm{~V}}$ and ChAT-tTA/ TDP- $43^{\mathrm{M} 337 \mathrm{~V}}$ rats at the age of 60 days. To stop TDP- $43^{\mathrm{M} 337 \mathrm{~V}}$ from further expression in diseased rats in a timely manner, Dox was subcutaneously injected ( $4 \mathrm{mg} / \mathrm{kg} / \mathrm{d}$ for 5 days) and was simultaneously given in drinking water $(500 \mu \mathrm{g} / \mathrm{ml})$. Dox was maintained at $500 \mu \mathrm{g} / \mathrm{ml}$ in drinking water until diseased rats were terminated.

Behavioral tests and disease stages. Grip strength of forepaws and hind paws was measured with a grip-strength meter (Columbus Instruments). Mobility was monitored by an open-field activity assay (Med Associates), which measured the total distance a rat traveled within 20 minutes. Grip strength and mobility were used for determining disease onset and progression in rats. Disease onset was defined as unrecoverable reduction of travel distance or grip strength. Paralysis was defined as occurrence of dragging legs or as inability to retract legs individually. Disease end stages were defined as inability to retract 2 or more legs, as inability to right itself when a rat was placed on its side, or as a severe loss of body weight (>30\%).

Cresyl violet staining and stereological cell counting. Coronal sections of rat forebrain or cross sections of rat spinal cord were stained with cresyl violet for cell counting. Total neurons in the dentate gyrus of 1 hemisphere were estimated by unbiased stereological cell counting. Every 12th section $(20 \mu \mathrm{m})$ of the dentate gyrus was counted for neurons (a total of 17 to 22 sections counted). To increase the accuracy of cell counting, a long segment of lumbar cord (L3-L5) was assessed for motor neurons. The lumbar cord was cut into consecutive sections $(30 \mu \mathrm{m})$, and every tenth section was counted for motor neurons on both sides of L3-L5 cords (a total of 15 to 20 sections counted). Stereological cell counting was done as described previously $(3,31)$.

Toluidine blue staining and electromicroscopy. As described previously $(3,31)$, the ultrastructure of axons in L3 ventral and dorsal roots was assessed by electromicroscopy and by toluidine blue staining. Deeply anesthetized rats were perfused with a mixture of $4 \%$ PFA and $2 \%$ glutaraldehyde. Nerve roots were dissected and post-fixed in the same fixative at $4^{\circ} \mathrm{C}$ overnight. Fixed tissues were embedded in Epon 812 and cut into semithin $(1 \mu \mathrm{m})$ and thin $(80 \mathrm{~nm})$ sections. Semithin sections stained with $1 \%$ toluidine blue were examined under a light microscope, and thin sections stained with uranyl acetate and lead citrate were examined under a transmission electron microscope.

$X$-gal staining and silver staining. Deeply anesthetized rats were perfused with $4 \%$ PFA for 2 hours. Lumbar cord was dissected and cut into cross sections of $30 \mu \mathrm{m}$ on a Cryostat. At room temperature, tissue sections were stained for 24 hours in PBS solution composed of $1 \mathrm{mg} / \mathrm{ml}$ of 5-bromo-4-chloro-3-indolyl- $\beta$-D-galactopyranoside (X-gal), $5 \mathrm{mM}$ potassium ferricyanide, $5 \mathrm{mM}$ potassium ferrocyanide, $2 \mathrm{mM} \mathrm{MgCl}_{2}, 0.02 \%$ NP-40, and $0.01 \%$ sodium deoxycholate. Sections stained with X-gal were counterstained with fast red. As described previously (3), degenerating neurons in spinal cord were detected by Bielschowsky silver staining on paraffin-embedded sections $(10 \mu \mathrm{m})$.

Histology and histochemistry in skeletal muscles. As described previously (3, 31), the structure of gastrocnemius muscle was assessed by H\&E staining and by histochemistry for nonspecific esterase and ATPase. Fresh muscle was snap-frozen in isopentane/liquid nitrogen and was cut into cross sections $(10 \mu \mathrm{m})$ on a Cryostat. Nonspecific esterase activity was detected using $\alpha$-napthyl acetate protocol. Myosin ATPase staining ( $\mathrm{pH}$ H.6) was employed to show 3 types of skeletal muscle fibers. Regenerated muscle usually presents as accumulated fibers of the same type and of varied sizes.

Antibodies. The following primary antibodies were used in this study: chicken anti-ubiquitin (Sigma-Aldrich), goat anti-ChAT (Millipore), guinea pig
anti-GLT1 (Millipore), mouse monoclonal anti-APC (Calbiochem), mouse monoclonal anti- $\beta$-gal (clone GAL-40; Sigma-Aldrich), mouse monoclonal anti-GAPDH (Abcam), mouse monoclonal anti-human TDP-43 (Abnova, clone 2E2-D3), rabbit anti-GFAP (DAKO North America), and rabbit antiIba-1 (Wako Chemicals USA). Polyclonal antibody against NeuN was raised in rabbits with a peptide (MAQPYPPAQYPPC) and was affinity purified.

Immunoblotting, immunohistochemistry, and immunofluorescence staining. As described previously (3), the expression levels of human TDP-43 in transgenic tissues were estimated by immunoblotting. The same membrane was incubated first with antibody to human TDP-43 and then with antibody to GAPDH after stripping. Forebrain and spinal cord were cut into coronal or cross sections on a Cryostat, respectively. Immunohistochemistry was done for human TDP-43, rat GFAP, rat Iba-1, rat ubiquitin, and rat NG2. Immunostained sections were visualized using an ABC Kit in combination with diaminobenzidine (Vector). Some immunostained sections were counterstained lightly with hematoxylin to display nuclei. Doublelabel immunofluorescence staining was used to show the colocalization of human TDP-43 with rat GFAP, rat NeuN, rat APC, rat ChAT, rat ubiquitin, or transgenic LacZ. Immunofluorescence staining and confocal microscopy were done as described previously $(3,31)$.

Quantitative PCR. Transgene copy and gene expression at the mRNA level were determined by quantitative PCR. The copy number of the tTA transgene was determined by quantitative PCR, as described in our publication (29). Expression of both human and rat TDP-43 mRNA was assessed by quantitative PCR with 2 pairs of primers. The first pair of primers was designed to amplify a fragment common to both human and rat TDP-43 mRNA: 5'-TGAGCCCATTGAAATACCATC-3' (forward) and 5'-TACACTGAGACACTGGATTC-3' (reverse). The second pair of primers was designed to amply the rat $L 17$ gene: $5^{\prime}$-TGGTTCGCTACTCCCTTGAC-3' (forward) and $5^{\prime}$-CTTGATGGCCTGGGCAGTT-3' (reverse). TDP-43 mRNA levels were first normalized to $L 17 \mathrm{mRNA}$, and the fold change of expression levels in transgenic cells was calculated relative to that of nontransgenic cells.

Statistics. The numbers of hippocampal neurons and spinal motor neurons were compared between groups of rats with different genotypes, and statistical differences in the numbers were analyzed by unpaired $t$ test. $P<0.05$ was considered significant.

Study approval. Animal use was in accord with NIH guidelines, and the animal use protocol was approved by the Institutional Animal Care and Use Committees at Thomas Jefferson University.

\section{Acknowledgments}

This work is supported by the NIH/National Institute of Neurological Disorders and Stroke (NS073829 to H. Zhou and NS072696, NS064042 and NS072113 to X.-G. Xia). The content is the authors' responsibility and does not necessarily represent the official view of the NIH. We would like to thank Xiaotao Wei, Dian Wang, and Zhen Qu for technical assistance. We also thank Qilin Cao for helping us with immunopanning.

Received for publication August 24, 2011, and accepted in revised form November 2, 2011.

Address correspondence to: Hongxia Zhou, Department of Pathology, Thomas Jefferson University, 1020 Locust Street, Philadelphia, Pennsylvania 19107, USA. Phone: 215.503.5916; Fax: 215.923.3808; E-mail: Hongxia.zhou@jefferson.edu. Or to: Xu-Gang Xia, Department of Pathology, Anatomy and Cell Biology, Thomas Jefferson University, 1020 Locust Street, Philadelphia, Pennsylvania 19107, USA. Phone: 215.503.9152; Fax: 215.503.7848; E-mail: xugang. xia@jefferson.edu. 
1. Sreedharan J, et al. TDP-43 mutations in familial and sporadic amyotrophic lateral sclerosis. Science. 2008;319(5870):1668-1672

2. Neumann M, et al. Ubiquitinated TDP-43 in frontotemporal lobar degeneration and amyotrophic lateral sclerosis. Science. 2006;314(5796):130-133.

3. Zhou $\mathrm{H}$, et al. Transgenic rat model of neurodegeneration caused by mutation in the TDP gene. PLoS Genet. 2010;6(3):e1000887.

4. Wegorzewska I, Bell S, Cairns NJ, Miller TM, Baloh RH. TDP-43 mutant transgenic mice develop features of ALS and frontotemporal lobar degeneration. Proc Natl Acad Sci US A. 2009;106(44):18809-18814.

5. Stallings NR, Puttaparthi K, Luther CM, Burns DK Elliott JL. Progressive motor weakness in transgenic mice expressing human TDP-43. NeurobiolDis. 2010; 40(2):404-414.

6. Wils $\mathrm{H}$, et al. TDP-43 transgenic mice develop spastic paralysis and neuronal inclusions characteristic of ALS and frontotemporal lobar degeneration. Proc Natl Acad Sci U S A. 2010;107(8):3858-3863.

7. Igaz LM, et al. Dysregulation of the ALS-associated gene TDP-43 leads to neuronal death and degeneration in mice. J Clin Invest. 2011;121(2):726-738.

8. Shan X, Chiang PM, Price DL, Wong PC. Altered distributions of Gemini of coiled bodies and mitochondria in motor neurons of TDP-43 transgenic mice. Proc Natl Acad Sci U S A. 2010;107(37):16325-16330.

9. Tsai KJ, et al. Elevated expression of TDP-43 in the forebrain of mice is sufficient to cause neurological and pathological phenotypes mimicking FTLD-U. J Exp Med. 2010;207(8):1661-1673

10. Benajiba L, et al. TARDBPmutations in motoneuron disease with frontotemporal lobar degeneration. Ann Neurol. 2009;65(4):470-473.

11. Buratti E, Dork T, Zuccato E, Pagani F, Romano M, Baralle FE. Nuclear factor TDP-43 and SR proteins promote in vitro and in vivo CFTR exon 9 skipping. EMBO J. 2001;20(7):1774-1784.

12. Bosco DA, et al. Mutant FUS proteins that cause amyotrophic lateral sclerosis incorporate into stress granules. Hum Mol Genet. 2010;19(21):4160-4175.

13. Wang I, Wu L, Shen C. TDP-43: an emerging new player in neurodegenerative diseases. Trends $\mathrm{Mol}$ Med. 2008;14(11):479-485.

14. Elden AC, et al. Ataxin-2 intermediate-length polyglutamine expansions are associated with increased risk for ALS. Nature. 2010;466(7310):1069-1075.

15. Polymenidou $\mathrm{M}$, et al. Long pre-mRNA depletion and RNA missplicing contribute to neuronal vulnerability from loss of TDP-43. Nat Neurosci. 2011; 14(4):459-468

16. Tollervey JR, et al. Characterizing the RNA targets and position-dependent splicing regulation by TDP-43. Nat Neurosci. 2011;14(4):452-458.

17. Rothstein JD, et al. Beta-lactam antibiotics offer neuroprotection by increasing glutamate trans- porter expression. Nature. 2005;433(7021):73-77.

18. Wang $\mathrm{H}$, et a;. Therapeutic gene silencing delivered by a chemically modified small interfering RNA against mutant SOD1 slows amyotrophic lateral sclerosis progression. J Biol Chem. 2008; 283(23):15845-15852.

19. Raoul C, et al. Lentiviral-mediated silencing of SOD1 through RNA interference retards disease onset and progression in a mouse model of ALS Nat Med. 2005;11(4):423-428.

20. Ralph GS, et al. Silencing mutant SOD1 using RNAi protects against neurodegeneration and extends survival in an ALS model. Nat Med. 2005; 11(4):429-433.

21. Williamson TL, Cleveland DW. Slowing of axonal transport is a very early event in the toxicity of ALSlinked SOD1 mutants to motor neurons. Nat Neu rosci. 1999;2(1):50-56.

22. Gu X, et al. Pathological cell-cell interactions elicited by a neuropathogenic form of mutant Huntingtin contribute to cortical pathogenesis in HD mice. Neuron. 2005;46(3):433-444.

23. Ko DC, Milenkovic L, Beier SM, Manuel H, Buchanan J, Scott MP. Cell-autonomous death of cerebellar purkinje neurons with autophagy in NiemannPick type C disease. PLoS Genet. 2005;1(1):81-95.

24. Custer SK, et al. Bergmann glia expression of polyglutamine-expanded ataxin-7 produces neurodegeneration by impairing glutamate transport. Nat NeuroSci. 2006;9(10):1302-1311.

25. Wang $\mathrm{L}$, et al. Restricted expression of mutant SOD1 in spinal motor neurons and interneurons induces motor neuron pathology. Neurobiol Dis. 2008;29(3):400-408.

26. Boillee S. Onset and progression in inherited ALS determined by motor neurons and microglia. Science. 2006;312(5778):1389-1392

27. Gurney ME, et al. Motor neuron degeneration in mice that express a human $\mathrm{Cu}, \mathrm{Zn}$ superoxide dismutase mutation. Science. 1994;264(5166):1772-1775.

28. Mackenzie IR, et al. Pathological TDP-43 distinguishes sporadic amyotrophic lateral sclerosis from amyotrophic lateral sclerosis with SOD1 mutations. Ann Neurol. 2007;61(5):427-434.

29. Zhou H, et al. Developing tTA transgenic rats for inducible and reversible gene expression. Int J Bio Sci. 2009;2(4):171-181.

30. Hirasawa M, Cho A, Sreenath T, Sauer B, Julien JP, Kulkarni AB. Neuron-specific expression of Cre recombinase during the late phase of brain development. Neurosci Res. 2001;40(2):125-132.

31. Huang C, et al. FUS transgenic rats develop the phenotypes of Amyotrophic Lateral Sclerosis and frontotemporal lobar degeneration. PLoS Genet. 2011; 7(3):e1002011.

32. Tallini YN, et al. BAC transgenic mice express enhanced green fluorescent protein in central and peripheral cholinergic neurons. Physiol Genomics. 2006;27(3):391-397.

33. Wiese $\mathrm{S}$, et al. Isolation and enrichment of embryonic mouse motoneurons from the lumbar spinal cord of individual mouse embryos. Nat Protoc. 2010; 5(1):31-38.

34. Yamanaka K, et al. Astrocytes as determinants of disease progression in inherited amyotrophic lateral sclerosis. Nat Neurosci. 2008;11(3):251-253.

35. Boillee $\mathrm{S}$, et al. Onset and progression in inherited ALS determined by motor neurons and microglia. Science. 2006;312(5778):1389-1392.

36. Lobsiger CS, et al. Schwann cells expressing dismutase active mutant SOD1 unexpectedly slow disease progression in ALS mice. Proc Natl Acad Sci US A. 2009;106(11):4465-4470.

37. Rutherford NJ, et al. Novel mutations in TARDBP (TDP-43) in patients with familial Amyotrophic Lateral Sclerosis. PLoS Genet. 2008;4(9):e1000193.

38. Yokoseki A, et al. TDP-43mutation in familial amyotrophic lateral sclerosis. Ann Neurol. 2008; 63(4):538-542.

39. Van Deerlin VM, et al. TARDBP mutations in amyotrophic lateral sclerosis with TDP-43 neuropathology: a genetic and histopathological analysis. Lancet Neurol. 2008;7(5):409-416.

40. Xu YF, et al. Wild-type human TDP-43 expression causes TDP-43 phosphorylation, mitochondrial aggregation, motor deficits, and early mortality in transgenic mice. J Neurosci. 2010;30(32):10851-10859.

41. Lino MM, Schneider C, Caroni P. Accumulation of SOD1 mutants in postnatal motoneurons does not cause motoneuron pathology or motoneuron disease. J Neurosci. 2002;22(12):4825-4832.

42. Ravits J, Laurie P, Fan Y, Moore DH. Implications of ALS focality: rostral-caudal distribution of lower motor neuron loss postmortem. Neurology. 2007; 68(19):1576-1582.

43. Ravits J, Paul P, Jorg C. Focality of upper and lower motor neuron degeneration at the clinical onset of ALS. Neurology. 2007;68(19):1571-1575.

44. Bruijn LI, et al. ALS-linked SOD1 mutant G85R mediates damage to astrocytes and promotes rapidly progressive disease with SOD1-containing inclusions. Neuron. 1997;18(2):327-338.

45. Wong PC, et al. An adverse property of a familial ALS-linked SOD1 mutation causes motor neuron disease characterized by vacuolar degeneration of mitochondria. Neuron. 1995;14(6):1105-1116.

46. Gitcho MA, et al. TARDBP 3'-UTR variant in autopsy-confirmed frontotemporal lobar degeneration with TDP-43 proteinopathy. Acta Neuropathol. 2009;118(5):633-645.

47. Ling SC, et al. ALS-associated mutations in TDP43 increase its stability and promote TDP-43 complexes with FUS/TLS. Proc Natl Acad Sci U S A. 2010; 107(30):13318-13323 